\title{
The rhizosphere microbiome and plant health
}

\author{
Roeland L. Berendsen ${ }^{1}$, Corné M.J. Pieterse ${ }^{1,2}$ and Peter A.H.M. Bakker ${ }^{1}$ \\ ${ }^{1}$ Plant-Microbe Interactions, Department of Biology, Faculty of Science, Utrecht University, Padualaan 8, $3584 \mathrm{CH}$ Utrecht, \\ The Netherlands \\ ${ }^{2}$ Centre for BioSystems Genomics, PO Box 98, 6700 AB Wageningen, The Netherlands
}

\begin{abstract}
The diversity of microbes associated with plant roots is enormous, in the order of tens of thousands of species. This complex plant-associated microbial community, also referred to as the second genome of the plant, is crucial for plant health. Recent advances in plantmicrobe interactions research revealed that plants are able to shape their rhizosphere microbiome, as evidenced by the fact that different plant species host specific microbial communities when grown on the same soil. In this review, we discuss evidence that upon pathogen or insect attack, plants are able to recruit protective microorganisms, and enhance microbial activity to suppress pathogens in the rhizosphere. A comprehensive understanding of the mechanisms that govern selection and activity of microbial communities by plant roots will provide new opportunities to increase crop production.
\end{abstract}

\section{The rhizosphere microbiome}

Pathogens can have a severe impact on plant health. The interactions between plants and pathogens are regularly simplified as trench warfare between the two parties, ignoring the importance of additional parties that can significantly affect the infection process. Plants live in close association with the microbes that inhabit the soil in which plants grow. Soil microbial communities represent the greatest reservoir of biological diversity known in the world so far [1-4]. The rhizosphere, which is the narrow zone of soil that is influenced by root secretions, can contain up to $10^{11}$ microbial cells per gram root [5] and more than 30,000 prokaryotic species [6]. The collective genome of this microbial community is much larger than that of the plant and is also referred to as the plant's second genome. In humans, the effects of intestinal microbial communities on health are becoming increasingly apparent [7]. Similar functions can be ascribed to microbial communities in the human gut and plant rhizosphere (Table 1). An increasing body of evidence also signifies the importance of this root microbiome, which consists of the entire complex of rhizosphere-associated microbes, their genetic elements and their interactions, in determining plant health. Here, we discuss how rhizosphere microbial communities, with an emphasis on bacteria, affect the plant and vice versa.
The root microbiome: effects on plant health

Disease-suppressive soils

The impact of the root microbiome on plant health is evidenced most clearly in disease-suppressive soils (Figure 1). The microflora of most soils is starved. As a result, there is a fierce battle in the rhizosphere between the microorganisms that compete for plant-derived nutrients [8]. Most soil-borne pathogens need to grow saprophytically in the rhizosphere to reach their host or to achieve sufficient numbers on their host before they can infect host tissue and effectively escape the rhizosphere battle zone. The success of a pathogen is influenced by the microbial community of the soil in which the infection takes place. Every natural soil has the ability to suppress a pathogen to a certain extent. This can be deduced from the disease severity following pathogen inoculation in pasteurized soils compared with non-pasteurized soils. This phenomenon is known as general disease suppression and is attributed to the total microbial activity. Organic amendments can stimulate the activity of microbial populations in a conducive soil, resulting in enhanced general disease suppressiveness [9]. 'Specific suppression' occurs when specific microorganisms cause soils to be suppressive to a disease $[8,10,11]$. Specific disease suppressiveness is superimposed on the general disease suppressiveness of soils and is more effective. The biotic nature of specific disease suppressiveness is also demonstrated by the removal of suppressiveness through pasteurization of the soil, but is distinguished from general suppressiveness because specific suppressiveness can be transferred to disease conducive soil by adding $0.1-10 \%$ of the suppressive soil $[6,8,10,11]$.

\section{Build-up of disease suppressiveness}

A further differentiation is made among specific diseasesuppressive soils; some soils retain their disease suppressiveness for prolonged periods and persist even when soils are left bare, whereas other soils develop suppressiveness only after monoculture of a crop for several years. Induction of suppressiveness by itself is remarkable, because for most plant species, successive monocultures will lead to a build-up of specialized plant pathogens [12]. Nonetheless, development of disease suppressiveness in soils has been reported for various diseases, including potato scab disease caused by Streptomyces species, Fusarium wilt disease of several plant species, Rhizoctonia damping-off disease of sugar beet, and take-all disease of wheat (Triticum aestivum) caused by Gaeumannomyces graminis var. tritici [6,8,11]. Microorganisms that can confer suppressiveness 
Table 1. Similarities of the microbiomes of the human gut and plant roots

\begin{tabular}{|c|c|c|c|c|}
\hline Characteristic & The human gut microbiome & Refs & The rhizosphere microbiome & Refs \\
\hline $\begin{array}{l}\text { Prevent colonization } \\
\text { by pathogens }\end{array}$ & $\begin{array}{l}\text { Mechanisms include competition for nutrients or } \\
\text { for adhesion receptors, production of inhibitory } \\
\text { metabolites and modulation of toxin production or } \\
\text { action }\end{array}$ & [86] & $\begin{array}{l}\text { Mechanisms include competition for } \\
\text { (micro)nutrients, production of antibiotic } \\
\text { compounds or lytic enzymes and consumption } \\
\text { of pathogen stimulatory compounds }\end{array}$ & {$[13,14]$} \\
\hline $\begin{array}{l}\text { Modulate host } \\
\text { immunity }\end{array}$ & $\begin{array}{l}\text { Host innate immune systems adapt to colonization } \\
\text { by microflora and shift to a primed state that not } \\
\text { only affects the intestinal mucosa, but can also } \\
\text { regulate immune response in respiratory mucosa } \\
\text { Furthermore, development of the gut microflora in } \\
\text { the first year of life is of crucial importance in the } \\
\text { development of the immune system and for } \\
\text { susceptibility to development of disease later in life }\end{array}$ & {$[7,86,88]$} & $\begin{array}{l}\text { Many beneficial soil-borne microorganisms } \\
\text { have been found to boost systemically the } \\
\text { defensive capacity of the plant. This ISR is a } \\
\text { state in which the plant immune system is } \\
\text { primed for accelerated activation of defense }\end{array}$ & [27] \\
\hline & $\begin{array}{l}\text { Many commensal microbes are potentially } \\
\text { pathogenic but are controlled by the host immune } \\
\text { system; commensals can become pathogenic in } \\
\text { immunity-impaired mice }\end{array}$ & & $\begin{array}{l}\text { Both pathogens and beneficials are also known } \\
\text { to suppress plant immune response to } \\
\text { promote their own colonization through } \\
\text { secretion of effector molecules }\end{array}$ & \\
\hline & $\begin{array}{l}\text { There is an indication that intestinal microbial } \\
\text { variation between individuals is stratified rather } \\
\text { than continuous, and that there is a limited amount } \\
\text { of classifiable communities that can exist in the } \\
\text { gut, coined 'enterotypes' }\end{array}$ & & $\begin{array}{l}\text { Root microbiomes of plants grown in the same } \\
\text { soil differ between plant species and between } \\
\text { genotypes within a species. However, the } \\
\text { existence of classifiable 'rhizotypes' has not yet } \\
\text { been reported }\end{array}$ & \\
\hline
\end{tabular}

to otherwise conducive soils have been isolated from many suppressive soils. Take-all, an important root disease of wheat caused by $G$. graminis var. tritici spontaneously decreases after several years of monoculture of wheat and a severe outbreak of the disease (Figure 1). This phenomenon is known as 'take-all decline'. It is observed worldwide and has been associated with the build-up of antagonistic fluorescent Pseudomonas spp. that produce the antifungal compound 2,4-diacetylphloroglucinol (DAPG) [11]. Other microorganisms that can confer suppressiveness have been found among the Proteobacteria and Firmicutes and for fungi among the Ascomycota (reviewed in [8]). Mechanisms through which rhizosphere microorganisms can affect a soil-borne pathogen have been identified and include production of antibiotic compounds, consumption of pathogen stimulatory compounds, competition for (micro)nutrients and production of lytic enzymes $[13,14]$.

\section{Modulation of the host immune system by beneficial microbes in the rhizosphere}

In addition to direct effects on deleterious microbes in the rhizosphere, many beneficial soil-borne microorganisms have been found to boost the defensive capacity in aboveground parts of the plant [15]. This induced systemic resistance (ISR) is a state in which the immune system of the plant is primed for accelerated activation of defense 


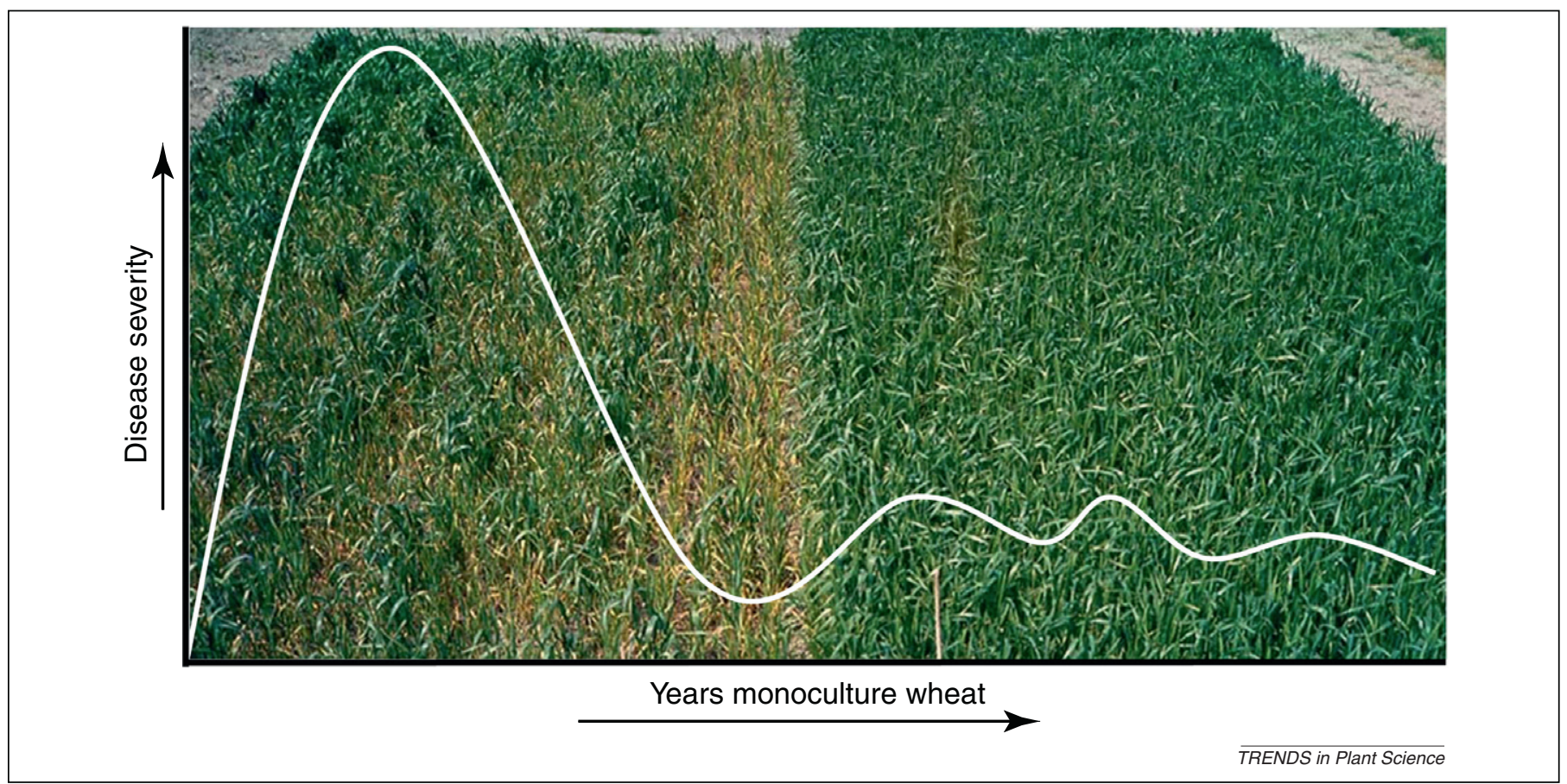

Figure 1. Disease-suppressive soils. For many soil-borne plant diseases, it has been found that the incidence of disease in some soils is lower than in surrounding soils, even though a virulent pathogen is present. Such disease-suppressive soils are especially well studied for take-all of wheat caused by Gaeumannomyces graminis var. tritici. During monoculture of wheat, take-all disease usually develops as depicted. An initial increase of disease severity as pathogen inoculum builds up with recurring presence of a susceptible host is followed by a decline of disease severity. This is typically associated with an increase of $P$ seudomonas spp. that produce the antifungal 2,4 diacetylphloroglucinol. The picture in the background shows an experimental field (Flevoland, the Netherlands) in which wheat had been grown in rotation with other crops (left) or in monoculture (right). Following inoculation with G. graminis var. tritici, less disease developed in the wheat monoculture plot. Adapted from [11].

[16-20]. Induction of ISR in Arabidopsis (Arabidopsis thaliana) by the plant growth-promoting rhizobacterium Pseudomonas fluorescens WCS417 is well studied. Locally in roots, WCS417 is able to suppress flagellin-triggered immune responses via apoplastic secretion of one or more low-molecular-weight molecules [21]. Although locally plant immunity is suppressed, an immune signaling cascade is initiated systemically that confers resistance against a broad spectrum of pathogens and even insects [22-24]. Initiation of this ISR in the roots of Arabidopsis is regulated by the root-specific transcription factor MYB72, which acts locally in the generation or translocation of a so far unknown systemic signal [25]. The ISR response is often not associated with direct defense activation, but instead with priming for accelerated defense-related gene expression and increased deposition of callose at the site of pathogen entry [23]. Establishment of WCS417-ISR in systemic leaf tissues depends on the hormones jasmonic acid and ethylene and requires the transcriptional (co)activators NPR1 and MYC2 ([26,27]. In addition to plant growth-promoting rhizobacteria, beneficial fungi such as mycorrhizal fungi [18], Trichoderma spp. [28] and other fungal biocontrol agents [29] have also been found to induce ISR. As well as inducing systemic resistance, mycorrhizal fungi can also form a connecting network between plants that can convey a resistance-inducing signal to neighboring plants [30].

\section{Microbiome complexity}

The identification of specific microorganisms responsible for disease suppressiveness has relied mainly on cultivationdependent techniques. Recently, bacterial communities have been characterized [6] in the rhizosphere of sugar beet grown in Rhizoctonia solani-suppressive soils using a highdensity $16 \mathrm{~S}$ ribosomal DNA oligonucleotide microarray referred to as PhyloChip [31]. More than 33,000 bacterial and archaeal operational taxonomic units were present in the rhizospheres of plants grown in either suppressive or conducive soil. However, neither the number nor the exclusive presence of microbial taxa could be related to disease suppressiveness. Instead, the relative abundance of several taxa was found to correspond to disease suppressiveness. The culture-independent approach identified the Gammaproteobacteria, Betaproteobacteria and Firmicutes as groups of bacteria that are important in disease suppressiveness. It was concluded that disease suppressiveness in the $R$. solani suppressive soil could not be attributed to a single taxon, but that it was brought about by a consortium of microorganisms.

Although specific microorganisms are able to protect the plant either directly or indirectly against pathogens, their efficacy is largely influenced by the rest of the microbial community. First, the pathogen-suppressing microorganisms should be present in sufficiently high numbers to have a significant effect $[32,33]$. Second, microorganisms that are regarded as commensals, because they neither harm nor benefit the plant directly, can compete effectively with the pathogen-suppressing biocontrol bacteria. Biocontrol bacteria may also act synergistically on each other, as seemingly non-antagonistic bacterial strains can become antagonistic when grown together with other specific strains [34]. Also, it was found that the soil-inhabiting $P$. fluorescens strain PF0-1 fine-tuned its transcriptional and metabolic responses in confrontation with different 
bacterial competitors and responded differently toward different species [35]. Conversely, negative effects of pathogens on their antagonists have also been reported. For example, Fusarium oxysporum produces fusaric acid, which downregulates the production of the antibiotic compound DAPG, a key factor in the antagonistic activity of $P$. fluorescens CHA0 against this pathogen [36]. It can be argued that all the active microorganisms affect each other in one way or another, albeit indirectly. Hence, although specific functions can be attributed to specific microorganisms, it is the total microbiome and its interactions that affect plant health (Figure 2).

\section{Plants actively shape their root microbiome Species-specific microbiomes}

The microflora of most soils is carbon starved [37]. Because plants secrete up to $40 \%$ of their photosynthates into the rhizosphere [38], the microbial population densities in the rhizosphere are much higher than in the surrounding bulk soil. This phenomenon is known as the 'rhizosphere effect'. In general, rhizosphere microbial communities are less diverse than those of the bulk soil [39-41]. It appears that, from the reservoir of microbial diversity that the bulk soil comprises, plant roots select for specific microorganisms to prosper in the rhizosphere. Together with the plant

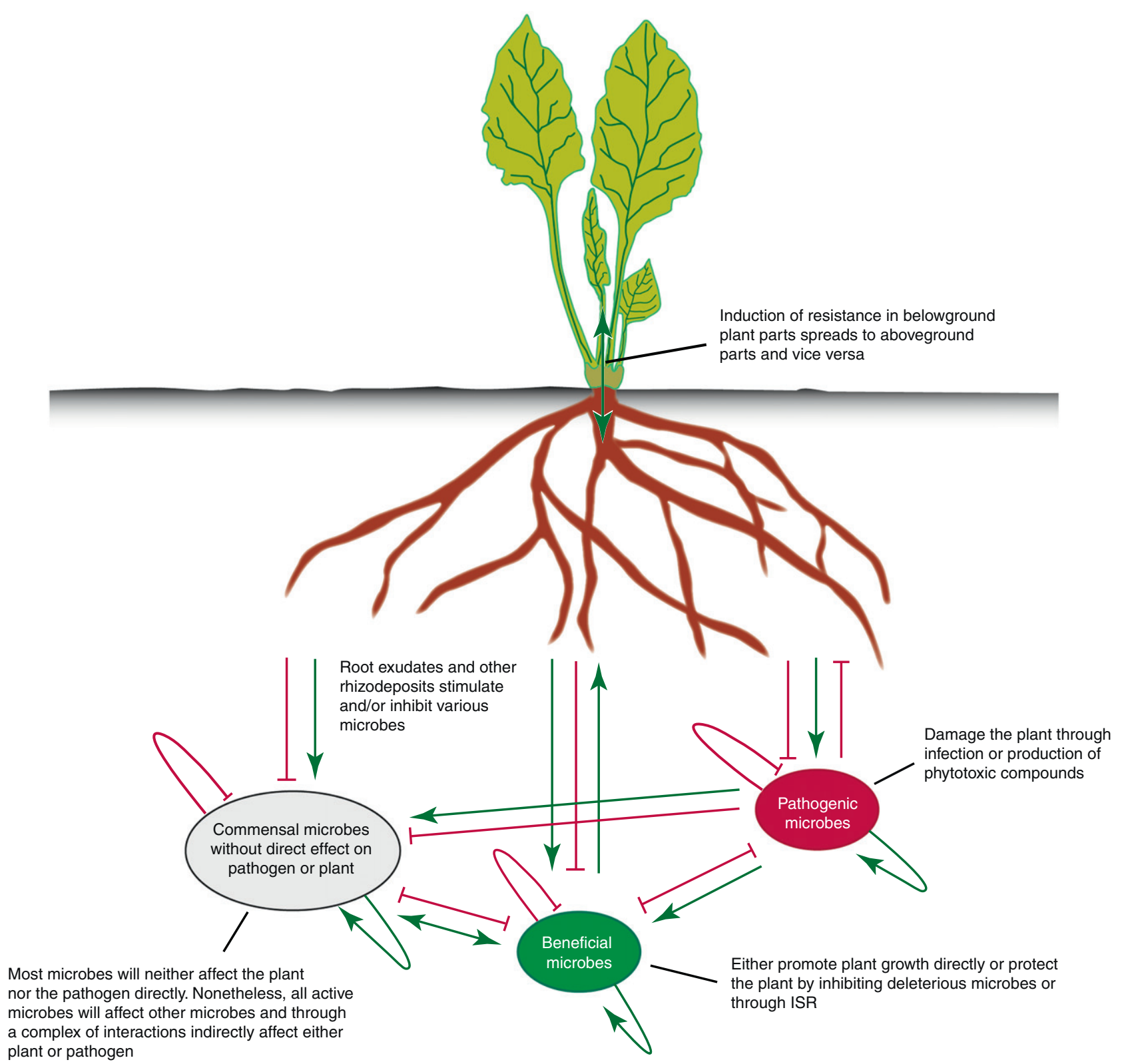

$\overline{\text { TRENDS in Plant Science }}$

Figure 2. Interactions in the rhizosphere. Plants are able to influence the composition and activation of their rhizosphere microbiome through exudation of compounds that stimulate (green arrows) or inhibit (red blocked arrows). Vice versa, a wide range of soil-borne pathogens are able to affect plant health. Prior to infection, these deleterious microbes are in competition with many other microbes in the rhizosphere for nutrients and space. In this battle for resources, beneficial microbes will limit the success of the pathogen through production of biostatic compounds, consumption of (micro)nutrients or by stimulating the immune system of the plant. Most microbes might neither affect the plant nor the pathogen directly because they occupy different ecological niches (commensal microbes), but are likely to affect every other organism to a certain extent through a complex network of interactions. Abbreviation: ISR, induced systemic resistance. 
genotype, soil type is an important driver of the microbial community composition in the rhizosphere $[42,43]$. However, microbial communities in the rhizospheres of different plant species growing on the same soil are also often different $[41,43,44]$; it has even been demonstrated that some plant species can create similar communities in different soils [45]. Even within species, different genotypes can develop distinct microbial communities in the rhizosphere [46], suggesting that plants are able to shape the composition of the microbiome in their rhizosphere.

\section{Microbiome management by the plant}

Plants can determine the composition of the root microbiome by active secretion of compounds that specifically stimulate or repress members of the microbial community [13]. In axenically collected exudates of seed, seedlings and roots of tomato (Lycopersicon esculentum), cucumber (Cucumis sativus) and sweet pepper (Capsicum annuum), organic acids were predominant, with citric acid, succinic acid and malic acid being the most common [47]. The ability of rhizobacterial strains to grow in vitro on citric acid as the sole carbon source appeared to correlate with their rootcolonizing ability. This indicates that plant species can select bacteria through the production of specific root exudates. Stable isotope probing of plants grown under ${ }^{13} \mathrm{CO}_{2}$ revealed that bacteria assimilate root exudates [48]. Using DGGE community profiling, this study also demonstrated that exudate-consuming bacterial rhizosphere populations of four plants species were more distinct than populations that did not utilize the root exudates, emphasizing the stimulatory role of root exudates in shaping the microbiome of a plant. Plant roots can also secrete secondary metabolites that inhibit growth of specific microbes in the rhizosphere $[49,50]$. For instance, benzoxazinoids are exuded in relatively large quantities from cereal roots and can inhibit rhizosphere microbes. In maize (Zea mays), 2,4-dihydroxy7-methoxy-2H-1,4-benzoxazin-3(4H)-one (DIMBOA) is the main antimicrobial benzoxazinoid. Interestingly, the plantbeneficial rhizobacterium Pseudomonas putida KT2440 is not only relatively tolerant to DIMBOA, but is also chemotactically attracted by this compound [51]. Roots of DIMBOA-deficient maize mutants were significantly less colonized by KT2440 than were wild-type plants, indicating that antimicrobial DIMBOA selectively attracts this plantbeneficial bacterium.

Furthermore, plant-associated bacteria produce and utilize diffusible $N$-acyl-homoserine lactones (AHLs) to signal to each other and to regulate their gene expression [52]. Such cell-to-cell communication is known as 'quorum sensing' (QS). AHL-mediated regulation typically makes use of two proteins that resemble the LuxI and LuxR protein families. LuxI-like proteins are AHL synthases, whereas LuxR-like proteins function as receptors of AHL that can form complexes with AHL and that in turn can affect gene expression of QS-target genes [53]. Plants can produce compounds that stimulate or repress QS-regulated responses in bacteria. A study testing the effect of seedling extracts and seedling exudates of Medicago truncatula found 15-20 compounds that specifically stimulated or repressed responses in QS-reporter bacteria [54]. Similar compounds that affect bacterial QS have been found in pea (Pisum sativum), rice (Oryza sativum) and green algae (Chlamydomonas reinhardtii) [55-57]. These QS-interfering compounds enable the plant to manipulate gene expression in their bacterial communities. Some plantassociated bacteria have LuxR-like proteins that are stimulated by plant-derived signals, whereas they themselves do not produce AHLs [57]. Also, bacterially produced AHLs have been shown to affect root development of Arabidopsis [58] and have been suggested to elicit ISR in tomato [59], further highlighting the importance of AHLs in crosskingdom signaling in the rhizosphere.

\section{Plant genotype determines microbiome composition}

Recent evidence suggests that differences between plant genotypes in a single gene can have a significant impact on the rhizosphere microbiome. The production of a single exogenous glucosinolate significantly altered the microbial community on the roots of transgenic Arabidopsis [60]. Alphaproteobacteria and fungal communities were mainly impacted, as shown by denaturing gel gradient electrophoresis of specific amplified fragments of $16 \mathrm{~S}$ and $18 \mathrm{~S}$ rRNA genes. Furthermore, it has been reported that an $\mathrm{ABC}$ transporter mutant of Arabidopsis, abcg30, had root exudates with increased phenolic compounds and decreased sugars, which also resulted in a distinct root microbiome [61].

A study of bacterial rhizosphere communities in two different soils on three cultivars of potato using the PhyloChip technology detected 2432 operational taxonomic units in the potato rhizosphere, of which $40 \%$ had a site-specific abundance [62]. Only 9\% of the operational taxonomic units had a cultivar-dependent abundance in one or the other soil, whereas the abundance of $4 \%$ of the operational taxonomic units was cultivar dependent in both soils. These results underline not only the importance of the soil in determining rhizosphere communities, but also that some microbes have a particular affinity for certain plant genotypes. Interestingly, differences in abundance on the three cultivars were mainly observed for microbes belonging to the Pseudomonales, Streptomycetaceae and Micromonosporaceae, which are an order and two families of bacteria, respectively, that have been extensively studied for their ability to control plant pathogens. These results indicate that the plant genotype can affect the accumulation of microorganisms that help the plant to defend itself against pathogen attack. Indeed, differences have been found in the ability of wheat cultivars to accumulate naturally occurring DAPG-producing Pseudomonas spp., resulting in differences in disease suppressiveness $[63,64]$. In addition, the amount of antibiotics produced by specific biocontrol strains on roots has been found to differ between wheat cultivars [65]. Furthermore, specific wheat cultivars were reported to support specific biological control bacteria differentially, which further establishes that there is a degree of specificity in the interactions between plant genotype and the composition of their microbial community [63].

\section{The root microbiome to the rescue}

\section{Microbiome changes upon defense activation}

The interactions between a plant and its root microbiome might change when the plant is attacked. Recently, it was 
demonstrated that infection of citrus by Candidatus Liberibacter asiaticus, associated with Huanglongbing, drastically altered the composition of citrus rhizosphere communities [66]. Also, Verticillium dahliae infections affected the microbial composition of cotton rhizospheres [67]. Changes in rhizosphere composition upon infection might be the result of the induced excretion of antimicrobial compounds by infected roots. In hairy root cultures of sweet basil (Ocimum basilicum), Pythium infection elicited the secretion of rosmarinic acid, a caffeic acid ester with antimicrobial activity [49]. Infection of barley (Hordeum vulgare) roots by Fusarium graminum induced the exudation of phenolic compounds with antifungal activity [68].

However, infection does not only lead to secretion of pathogen-deterring compounds, for example infection of water melon plants by $F$. oxysporum enhanced the stimulation of Fusarium spore germination by root exudates [69]. In the same study, association of the biocontrol bacterium Paenibacillus polymyxa SQR-21 decreased the germination-stimulatory effects of the root exudates.

\section{Recruitment of beneficial microbes}

A study on foliar phloem feeding by white fly (Bemisia tabaci) on sweet pepper found that it elicited resistance against the bacterial root pathogen Ralstonia solanacearum [70]. Intriguingly, white fly feeding also led to significant changes in the rhizosphere microbial community. Although total numbers of bacteria were unaffected, the white flyinduced plants had higher populations of Gram-positive bacteria and fungi in their rhizosphere. The authors hypothesized that plants recruit plant-beneficial microbes to their roots in response to the attack (Figure 3 ). In line with this, aphid (Myzus persicae) feeding on pepper plants increased the root populations of the plant-beneficial Bacillus subtilis GB03, but reduced populations of the pathogenic $R$. solanacearum [71]. Recruitment of a beneficial bacterium has also been demonstrated for Arabidopsis when leaves were infected with a bacterial pathogen [72]. In this study, colonization of the roots of Arabidopsis by the plant-beneficial soil bacterium Bacillus subtilis FB17 was greatly improved when aboveground plant tissues were infected by Pseudomonas syringae pv. tomato. HPLC analyses of root exudates revealed increased secretion of malic acid upon $P$. syringae pv. tomato infection. The Bacillus strain was chemotactically attracted by malic acid, whereas other rhizobacteria were not. In addition, Atalmt1, an Arabidopsis knockout mutant deficient in root malic acid secretion, could no longer recruit FB17 after infection with $P$. syringae pv. tomato. Also, AtALMT1, which encodes a malic acid transporter, was found to be upregulated upon infection of the leaves by $P$. syringae pv. tomato. Colonization of Arabidopsis roots by FB17 induced ISR and protected the aerial parts of the

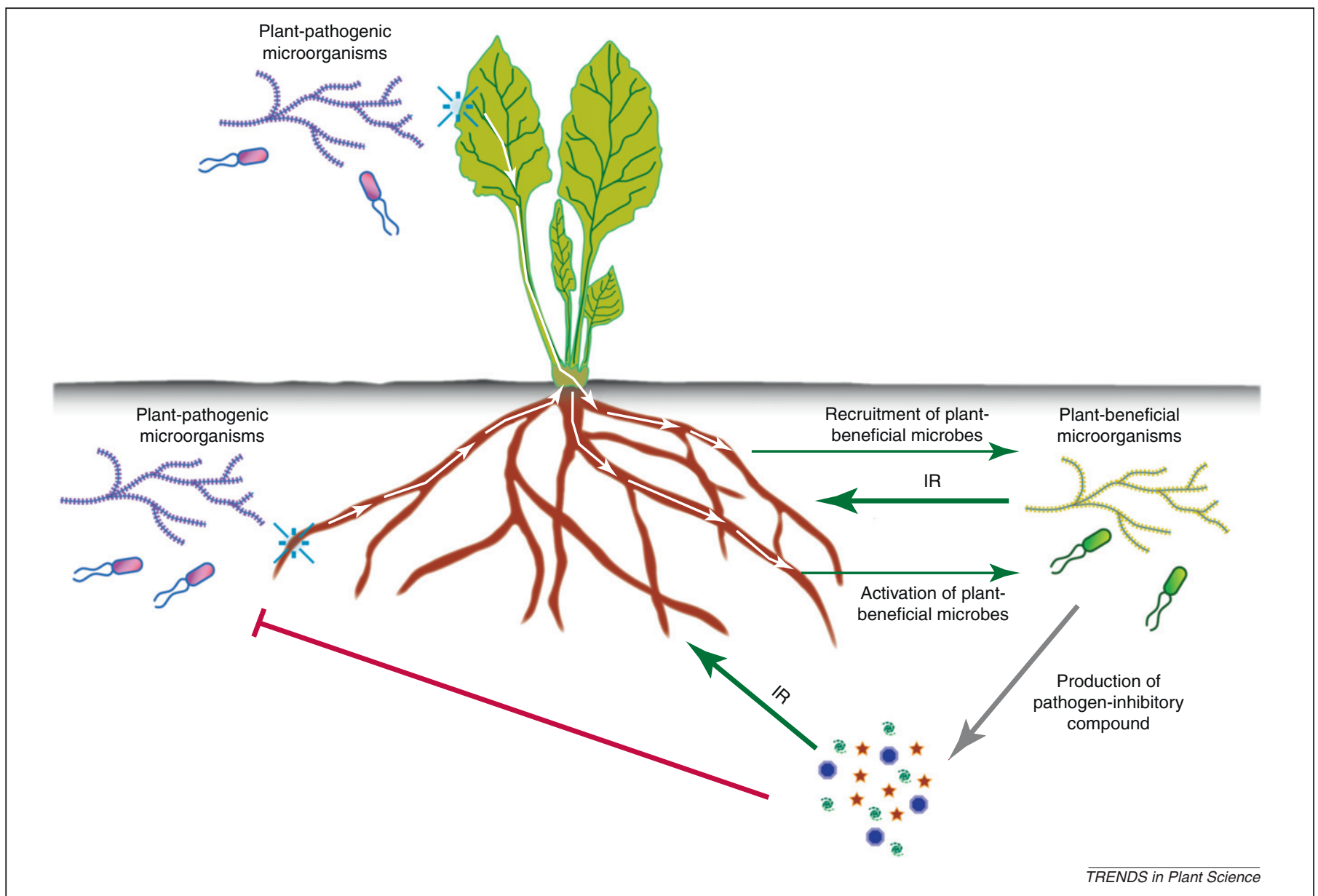

Figure 3. Microbiome to the rescue. Model of recruitment and activation of beneficials by the plant upon attack. Infected plants perceive pathogen invasion in roots or shoot and subsequently increase the secretion of microbe-stimulatory compounds in non-infected roots. These stimulants can recruit and activate plant-beneficial microorganisms. Beneficial microorganisms can induce resistance (IR) directly or produce pathogen-inhibitory compounds. Some pathogen-inhibitory compounds are known to induce resistance themselves [94]. 
plants against $P$. syringae pv. tomato infection. These data indicate a mutually beneficial relationship between Arabidopsis and FB17, whereby FB17 is recruited to aid in defense of the plant, and the plant provides the bacterium with malic acid.

In early studies, it was postulated that development of disease suppressiveness requires a severe outbreak of disease, after which plants signal for help in their rhizosphere [73]. Indeed, the above-described studies [71,72] indicate that plants respond to pathogen attack by recruiting specific beneficial microorganisms. It was recently reported that irrigated wheat fields in the pacific Northwest have high populations of Pseudomonas spp. that produce DAPG, whereas non-irrigated fields are dominated by Pseudomonas spp. that produce phenazines [74] In the irrigated fields, the DAPG-sensitive G. graminis var. tritici is the main root pathogen, whereas in the dry fields Rhizoctonia, which is sensitive to phenazine, is more problematic. This field study suggests specific recruitment of beneficial pseudomonads that are effective against a particular pathogen.

A study on shifts in the rhizosphere microflora of barley grown for five consecutive cycles in the same soil observed that barley induced shifts in the composition of the microbial communities [75]. However, the authors found no differences in microbiome composition between microcosms inoculated with the take-all fungus $G$. graminis var. tritici and non-inoculated microcosms. Nonetheless, take-all decline developed only in the inoculated microcosms. It was proposed that the major shifts in composition of microflora were a result of plant-specific selection of microbes, whereas the suppressiveness was a result of pathogen-induced changes in activity of the microbes present.

\section{Activation of beneficials}

The association of the pathogenic fungus $G$. graminis var. tritici with wheat roots strongly altered gene expression of the biocontrol bacterium P. fluorescens Pf29Arp [76]. Moreover, inoculation of strawberry plants with $V$. dahliae stimulated the expression of cyanide biosynthetic genes in the biocontrol bacterium Pseudomonas sp. LBUM300 in addition to stimulating root colonization by the bacterium [77]. These changes in gene expression could be a result of nutrients leaking from damaged roots. In a study of barley plants in a split root system, the roots on one side of the system were inoculated with Pythium ultimum, whereas the other side was inoculated with the biocontrol bacterium $P$. fluorescens CHA0 [78]. Using reporter strains, it was demonstrated that the expression of the DAPG biosynthesis gene phlA in CHA0 was induced upon infection by Pythium and that root exudation of vanillic acid, fumaric acid and $p$-coumaric acid increased concurrently. Very low concentrations of these organic acids can induce DAPG production in CHA0 in vitro. These results imply that, upon pathogen attack, the plant launches a systemic response that can stimulate the antifungal activity of the rhizosphere microflora (Figure 3).

\section{Effects of defense signaling on the root microbiome}

Several studies have investigated the effects of defense signaling on the commensal microflora. Differences have been observed in the microbial communities both in the phyllosphere [79] and in the rhizosphere $[40,80]$ of wildtype Arabidopsis plants and mutants impaired in defense signaling. In addition, defense-related plant hormones, such as jasmonic acid and salicylic acid, can mediate changes in the composition of root exudates [81]. This suggests that changes in the defense-related hormone signature, as observed during pathogen and insect attack of foliar tissues [82], potentially influence the composition of the root exudates and, hence, the composition of the microbiome in the rhizosphere. However, chemical activation of defense by foliar treatment with salicylic acid and jasmonic acid did not significantly affect the resident soil microflora [80]. Nonetheless, recruitment of beneficials upon defense activation probably involves specific interactions with only a small part of the microbiome and subtle changes in the composition of the microflora. Hence, development of specific and sensitive profiling techniques is crucial to detect such dynamic changes in the microbiome of a plant.

\section{Concluding remarks and prospects}

Current understanding of the complex plant-microbe interactions that take place in the rhizosphere is still in its infancy [83]. Experimental evidence underlines the importance of the root microbiome in plant health and it is becoming increasingly clear that the plant is able to control the composition of its microbiome. It stands to reason that those plants that manage their microbiome in a way that is beneficial to their reproductive success will be favored during evolutionary selection. It appears that such selective pressure has brought about many specific interactions between plants and microbes, and evidence is accumulating that plants call for microbial help in time of need. It is expected that the near future will bring many new insights into the selective forces that shape the microbiome of the root and how it affects the plant, because next-generation sequencing techniques will undoubtedly provide new opportunities to study the interplay between the plant and its associated microflora [84]. Metagenomic studies of the root microbiome have until now focused on phylogenetic composition, resulting in limited information on the presence of specific operational taxonomic units. Development of functional metagenomics and transcriptomics will deliver insight into the activities and functions of the microbiome. Ultimately, unraveling the mechanisms through which plants control their microbiome and through which the microbiome controls plant health will open new avenues to increase crop quality and productivity.

\section{Acknowledgments}

This work was financially supported by ERC Advanced Grant no. 269072 of the European Research Council.

\section{References}

1 Curtis, T.P. et al. (2002) Estimating prokaryotic diversity and its limits. Proc. Natl. Acad. Sci. U.S.A. 99, 10494-10499

2 Torsvik, V. et al. (2002) Prokaryotic diversity - magnitude, dynamics, and controlling factors. Science 296, 1064-1066

3 Buée, M. et al. (2009) 454 Pyrosequencing analyses of forest soils reveal an unexpectedly high fungal diversity. New Phytol. 184, 449-456

4 Gams, W. (2007) Biodiversity of soil-inhabiting fungi. Biodivers. Conserv. 16, 69-72 
5 Egamberdieva, D. et al. (2008) High incidence of plant growthstimulating bacteria associated with the rhizosphere of wheat grown on salinated soil in Uzbekistan. Environ. Microbiol. 10, 1-9

6 Mendes, R. et al. (2011) Deciphering the rhizosphere microbiome for disease-suppressive bacteria. Science 332, 1097-1100

7 Bron, P.A. et al. (2012) Emerging molecular insights into the interaction between probiotics and the host intestinal mucosa. Nat. Rev. Microbiol. 10, 66-78

8 Raaijmakers, J.M. et al. (2009) The rhizosphere: a playground and battlefield for soilborne pathogens and beneficial microorganisms. Plant Soil 321, 341-361

9 Hoitink, H. and Boehm, M. (1999) Biocontrol within the context of soil microbial communities: a substrate-dependent phenomenon. Annu. Rev. Phytopathol. 37, 427-446

10 Garbeva, P. et al. (2004) Microbial diversity in soil: selection of microbial populations by plant and soil type and implications for disease suppressiveness. Annu. Rev. Phytopathol. 42, 243-270

11 Weller, D.M. et al. (2002) Microbial populations responsible for specific soil suppressiveness to plant pathogens. Annu. Rev. Phytopathol. 40, 309-348

12 Bennett, A.J. et al. (2012) Meeting the demand for crop production: the challenge of yield decline in crops grown in short rotations. Biol. Rev. $87,52-71$

13 Doornbos, R. et al. (2012) Impact of root exudates and plant defense signaling on bacterial communities in the rhizosphere. A review. Agron. Sustain. Dev. 32, 227-243

14 Lugtenberg, B. and Kamilova, F. (2009) Plant-growth-promoting Rhizobacteria. Annu. Rev. Microbiol 63, 541-556

15 Zamioudis, C. and Pieterse, C.M.J. (2012) Modulation of host immunity by beneficial microbes. Mol. Plant Microbe Interact. 25, 139-150

16 Bakker, P.A.H.M. et al. (2007) Induced systemic resistance by fluorescent Pseudomonas spp. Phytopathology 97, 239-243

17 Conrath, U. et al. (2006) Priming: getting ready for battle. Mol. Plant Microbe Interact. 19, 1062-1071

18 Pozo, M.J. and Azcón-Aguilar, C. (2007) Unraveling mycorrhizainduced resistance. Curr. Opin. Plant Biol. 10, 393-398

19 De Vleesschauwer, D. and Höfte, M. (2009) Rhizobacteria-induced systemic resistance. In Plant Innate Immunity (Van Loon, L.C., ed.), pp. 223-281, Academic Press

20 Van Wees, S.C.M. et al. (2008) Plant immune responses triggered by beneficial microbes. Curr. Opin. Plant Biol. 11, 443-448

21 Millet, Y.A. et al. (2010) Innate immune responses activated in Arabidopsis roots by microbe-associated molecular patterns. Plant Cell 22, 973-990

22 Pineda, A. et al. (2010) Helping plants to deal with insects: the role of beneficial soil-borne microbes. Trends Plant Sci. 15, 507-514

23 Van der Ent, S. et al. (2009) Jasmonate signaling in plant interactions with resistance-inducing beneficial microbes. Phytochemistry 70, $1581-1588$

24 Van Oosten, V.R. et al. (2008) Differential effectiveness of microbially induced resistance against herbivorous insects in Arabidopsis. Mol. Plant Microbe Interact. 21, 919-930

25 Van der Ent, S. et al. (2008) MYB72 is required in early signaling steps of rhizobacteria-induced systemic resistance in Arabidopsis. Plant Physiol. 146, 1293-1304

26 Pozo, M.J. et al. (2008) Transcription factor MYC2 is involved in priming for enhanced defense during rhizobacteria-induced systemic resistance in Arabidopsis thaliana. New Phytol. 180, 511-523

27 Van der Ent, S. et al. (2009) Priming of plant innate immunity by rhizobacteria and $\beta$-aminobutyric acid: differences and similarities in regulation. New Phytol. 183, 419-431

28 Segarra, G. et al. (2009) MYB72, a node of convergence in induced systemic resistance triggered by a fungal and a bacterial beneficial microbe. Plant Biol. 11, 90-96

29 Shoresh, M. et al. (2010) Induced systemic resistance and plant responses to fungal biocontrol agents. Annu. Rev. Phytopathol. 48, 21-43

30 Song, Y.Y. et al. (2010) Interplant communication of tomato plants through underground common mycorrhizal networks. PLOS ONE 5, $1-11$

31 Hazen, T.C. et al. (2010) Deep-sea oil plume enriches Indigenous oildegrading bacteria. Science 330, 204-208
32 Raaijmakers, J.M. et al. (1995) Dose-response relationships in biological-control of Fusarium-wilt of radish by Pseudomonas spp. Phytopathology 85, 1075-1081

33 Bull, C.T. et al. (1991) Relationship between root colonization and suppression of Gaeumannomyces graminis var. tritici by Pseudomonas fluorescens Strain 2-79. Phytopathology 81, 954-959

34 De Boer, W. et al. (2007) In vitro suppression of fungi caused by combinations of apparently non-antagonistic soil bacteria. FEMS Microbiol. Ecol. 59, 177-185

35 Garbeva, P. et al. (2011) Transcriptional and antagonistic responses of Pseudomonas fluorescens Pf0-1 to phylogenetically different bacterial competitors. ISME J. 5, 1-13

36 Notz, R. et al. (2002) Fusaric acid-producing strains of Fusarium oxysporum alter 2,4-diacetylphloroglucinol biosynthetic gene expression in Pseudomonas fluorescens CHA0 in vitro and in the rhizosphere of wheat. Appl. Environ. Microbiol. 68, 2229-2235

37 Garbeva, P. et al. (2011) Fungistasis and general soil biostasis - a new synthesis. Soil Biol. Biochem. 43, 469-477

38 Bais, H.P. et al. (2006) The role of root exudates in rhizosphere interactions with plants and other organisms. Annu. Rev. Plant Biol. 57, 233-266

39 Costa, R. et al. (2006) Effects of site and plant species on rhizosphere community structure as revealed by molecular analysis of microbial guilds. FEMS Microbiol. Ecol. 56, 236-249

40 Hein, J. et al. (2008) Comparison of rizosphere bacterial communities in Arabidopsis thaliana mutants for systemic acquired resistance. Microb. Ecol. 55, 333-343

41 Berg, G. et al. (2006) The rhizosphere effect on bacteria antagonistic towards the pathogenic fungus Verticillium differs depending on plant species and site. FEMS Microbiol. Ecol. 56, 250-261

42 Berg, G. and Smalla, K. (2009) Plant species and soil type cooperatively shape the structure and function of microbial communities in the rhizosphere. FEMS Microbiol. Ecol. 68, 1-13

43 Garbeva, P. et al. (2008) Rhizosphere microbial community and its response to plant species and soil history. Plant Soil 302, 19-32

44 Viebahn, M. et al. (2005) Assessment of differences in ascomycete communities in the rhizosphere of field-grown wheat and potato. FEMS Microbiol. Ecol. 53, 245-253

45 Miethling, R. et al. (2000) Variation of microbial rhizosphere communities in response to crop species, soil origin, and inoculation with Sinorhizobium meliloti L33. Microb. Ecol. 40, 43-56

46 Micallef, S.A. et al. (2009) Influence of Arabidopsis thaliana accessions on rhizobacterial communities and natural variation in root exudates. J. Exp. Bot. 60, 1729-1742

47 Kamilova, F. et al. (2006) Organic acids, sugars, and l-tryptophane in exudates of vegetables growing on stonewool and their effects on activities of rhizosphere bacteria. Mol. Plant Microbe Interact. 19, 250-256

48 Haichar, F.e.Z. et al. (2008) Plant host habitat and root exudates shape soil bacterial community structure. ISME J. 2, 1221-1230

49 Bais, H.P. et al. (2002) Root specific elicitation and antimicrobial activity of rosmarinic acid in hairy root cultures of Ocimum basilicum. Plant Physiol. Biochem. 40, 983-995

50 Zhang, S.S. et al. (2011) Secondary metabolites from the invasive Solidago canadensis L. accumulation in soil and contribution to inhibition of soil pathogen Phytium ultimum. Appl. Soil Ecol. 48, 280-286

51 Neal, A.L. et al. (2012) Benzoxazinoids in root exudates of maize attract Pseudomonas putida to the rhizosphere. PLoS ONE 7, e35498 http:// dx.doi.org/10.1371/journal.pone.0035498

52 Elasri, M. et al. (2001) Acyl-homoserine lactone production is more common among plant-associated Pseudomonas spp. than among soilborne Pseudomonas spp. Appl. Environ. Microbiol. 67, 1198-1209

53 Decho, A.W. et al. (2011) Chemical challenges to bacterial AHL signaling in the environment. Chem. Rev. 111, 86-99

54 Gao, M. et al. (2003) Production of substances by Medicago truncatula that affect bacterial quorum sensing. Mol. Plant Microbe Interact. 16, 827-834

55 Teplitski, M. et al. (2000) Plants secrete substances that mimic bacterial $N$-acyl homoserine lactone signal activities and affect population density-dependent behaviors in associated bacteria. Mol. Plant Microbe Interact. 13, 637-648 
56 Teplitski, M. et al. (2004) Chlamydomonas reinhardtii secretes compounds that mimic bacterial signals and interfere with quorum sensing regulation in bacteria. Plant Physiol. 134, 137-146

57 Ferluga, S. and Venturi, V. (2009) OryR is a LuxR-family protein involved in interkingdom signaling between pathogenic Xanthomonas oryzae pv. oryzae and rice. J. Bacteriol. 191, 890-897

58 Ortiz-Castro, R. et al. (2008) $\mathrm{N}$-acyl-L-homoserine lactones: a class of bacterial quorum-sensing signals alter post-embryonic root development in Arabidopsis thaliana. Plant Cell Environ. 31, 1497-1509

59 Schuhegger, R. et al. (2006) Induction of systemic resistance in tomato by $N$-acyl-L-homoserine lactone-producing rhizosphere bacteria. Plant Cell Environ. 29, 909-918

60 Bressan, M. et al. (2009) Exogenous glucosinolate produced by Arabidopsis thaliana has an impact on microbes in the rhizosphere and plant roots. ISME J. 3, 1243-1257

61 Badri, D.V. et al. (2009) An ABC transporter mutation alters root exudation of phytochemicals that provoke an overhaul of natural soil microbiota. Plant Physiol. 151, 2006-2017

62 Weinert, N. et al. (2011) PhyloChip hybridization uncovered an enormous bacterial diversity in the rhizosphere of different potato cultivars: many common and few cultivar-dependent taxa. FEMS Microbiol. Ecol. 75, 497-506

63 Meyer, J.B. et al. (2010) Interplay between wheat cultivars, biocontrol pseudomonads, and soil. Appl. Environ. Microbiol. 76, 6196-6204

$64 \mathrm{Gu}, \mathrm{Y}-\mathrm{H}$. and Mazzola, M. (2003) Modification of fluorescent pseudomonad community and control of apple replant disease induced in a wheat cultivar-specific manner. Appl. Soil Ecol. 24, 57-72

65 Okubara, P.A. and Bonsall, R.F. (2008) Accumulation of Pseudomonasderived 2,4-diacetylphloroglucinol on wheat seedling roots is influenced by host cultivar. Biol. Control 46, 322-331

66 Trivedi, P. et al. (2012) Huanglongbing alters the structure and functional diversity of microbial communities associated with citrus rhizosphere. ISME J. 6, 363-383

67 Zhang, Y.et al. (2011) Analysis of bacterial communities in rhizosphere soil of healthy and diseased cotton (Gossypium sp.) at different plant growth stages. Plant Soil 339, 447-455

68 Lanoue, A. et al. (2010) De novo biosynthesis of defense root exudates in response to Fusarium attack in barley. New Phytol. 185, 577-588

69 Ling, N. et al. (2011) Paenibacillus polymyxa SQR-21 systemically affects root exudates of watermelon to decrease the conidial germination of Fusarium oxysporum f. sp. niveum. Plant Soil 341, 485-493

70 Yang, J.W. et al. (2011) Whitefly infestation of pepper plants elicits defence responses against bacterial pathogens in leaves and roots and changes the below-ground microflora. J. Ecol. 99, 46-56

71 Lee, B. et al. (2012) Foliar aphid feeding recruits rhizosphere bacteria and primes plant immunity against pathogenic and non-pathogenic bacteria in pepper. Ann. Bot. 110, 281-290

72 Rudrappa, T. et al. (2008) Root-secreted malic acid recruits beneficial soil bacteria. Plant Physiol. 148, 1547-1556

73 Cook, R.J.et al. (1995) Molecular mechanisms of defense by rhizobacteria against root disease. Proc. Natl. Acad. Sci. U.S.A. 92, 4197-4201

74 Mavrodi, O.V.et al. (2012) Irrigation differentially impacts populations of indigenous antibiotic-producing Pseudomonas spp. in the rhizosphere of wheat. Appl. Environ. Microbiol. 78, 3214-3220
75 Schreiner, K. et al. (2010) Comparison of barley succession and take-all disease as environmental factors shaping the rhizobacterial community during take-all decline. Appl. Environ. Microbiol. 76, 4703-4712

76 Barret, M. et al. (2009) Effect of wheat roots infected with the pathogenic fungus Gaeumannomyces graminis var. tritici on gene expression of the biocontrol bacterium Pseudomonas fluorescens Pf29Arp. Mol. Plant Microbe Interact. 22, 1611-1623

77 DeCoste, N.J. et al. (2010) Verticillium dahliae alters Pseudomonas spp. populations and $H C N$ gene expression in the rhizosphere of strawberry. Can. J. Microbiol. 56, 906-915

78 Jousset, A. et al. (2011) Plants respond to pathogen infection by enhancing the antifungal gene expression of root-associated bacteria. Mol. Plant Microbe Interact. 24, 352-358

79 Kniskern, J.M. et al. (2007) Salicylic acid and jasmonic acid signaling defense pathways reduce natural bacterial diversity on Arabidopsis thaliana. Mol. Plant Microbe Interact. 20, 1512-1522

80 Doornbos, R.F. et al. (2011) Effects of jasmonic acid, ethylene, and salicylic acid signaling on the rhizosphere bacterial community of Arabidopsis thaliana. Mol. Plant Microbe Interact. 24, 395-407

81 Badri, D.V. et al. (2008) Transcriptome analysis of Arabidopsis roots treated with signaling compounds: a focus on signal transduction, metabolic regulation and secretion. New Phytol. 179, 209-223

82 Pieterse, C.M.J. et al. (2009) Networking by small-molecule hormones in plant immunity. Nat. Chem. Biol. 5, 308-316

83 Bisseling, T. et al. (2009) Next-generation communication. Science 324,691

84 Schenk, P.M. et al. (2012) Unraveling plant-microbe interactions: can multi-species transcriptomics help? Trends Biotechnol. 30, 177-184

85 Derrien, M. et al. (2010) Mucin-bacterial interactions in the human oral cavity and digestive tract. Gut Microbes 1, 254-268

86 Fagundes, C.T. et al. (2012) Adapting to environmental stresses: the role of the microbiota in controlling innate immunity and behavioral responses. Immunol. Rev. 245, 250-264

87 Van Der Heijden, M.G.A. et al. (2008) The unseen majority: soil microbes as drivers of plant diversity and productivity in terrestrial ecosystems. Ecol. Lett. 11, 296-310

88 Ichinohe, T. et al. (2011) Microbiota regulates immune defense against respiratory tract influenza A virus infection. Proc. Natl. Acad. Sci. U.S.A. 108, 5354-5359

89 Chinen, T. and Rudensky, A.Y. (2012) The effects of commensal microbiota on immune cell subsets and inflammatory responses. Immunol. Rev. 245, 45-55

90 Lathrop, S.K. et al. (2011) Peripheral education of the immune system by colonic commensal microbiota. Nature 478, 250-254

91 Arumugam, M. et al. (2011) Enterotypes of the human gut microbiome. Nature 473, 174-180

92 Ley, R.E. et al. (2006) Ecological and evolutionary forces shaping microbial diversity in the human intestine. Cell 124, 837-848

93 Roesch, L.F.W. et al. (2007) Pyrosequencing enumerates and contrasts soil microbial diversity. ISME J. 1, 283-290

94 Weller, D.M. et al. (2012) Induced systemic resistance in Arabidopsis thaliana against Pseudomonas syringae pv. tomato by 2,4-diacetylphloroglucinol-producing Pseudomonas fluorescens. Phytopathology 102, 403-412 